\title{
Zwei Neue im AeDA-Vorstand
}

A uf dem 11. Deutschen Allergiekongress in Berlin wurden zwei Mitglieder neu in den AeDA-Vorstand gewählt - als Beisitzer sind hinzugekommen: Prof. Dr. Randolf Brehler, Münster, und Dr. Holger Wrede, Her-

ford. Die bewährte Vorstandsriege - mit Präsident Prof. Dr. Ludger Klimek, Vizepräsident Prof. Dr. Thomas Fuchs und Prof. Dr. Wolfgang W. Schlenter ist unverändert geblieben. Im Anschluss stellen sich die neuen Beisitzer kurz vor.

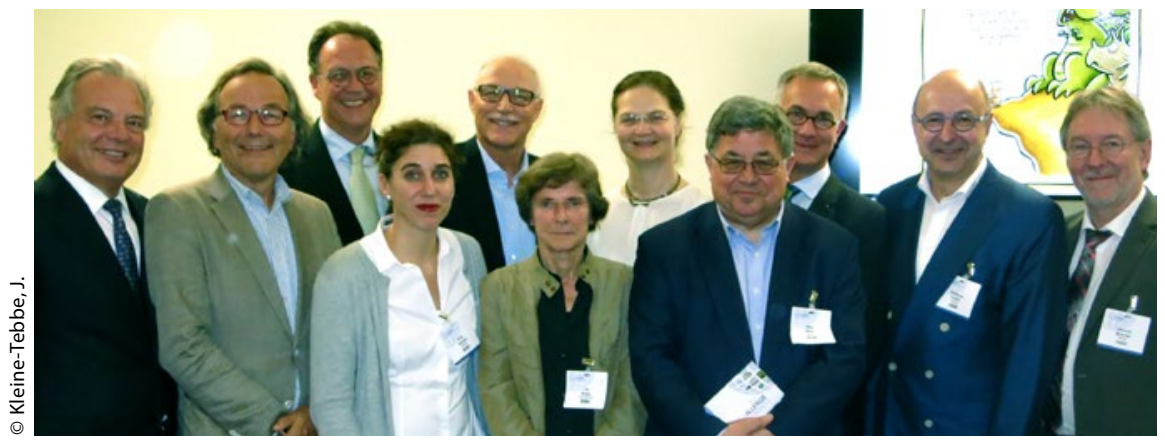

Der aktuelle AeDA-Vorstand - von links nach rechts: Prof Dr. Wolfgang Schlenter, Prof. Dr. Thomas Fuchs, Prof. Dr. Ludger Klimek, Dr. Katja Nemat, Prof. Dr. Wolfgang Wehrmann, Dr. Uta Rabe, PD Dr. Kirsten Jung; Prof. Dr. Hans Merk, Dr. Holger Wrede, Prof. Dr. Wolfgang Czech, Prof. Dr. Randolf Brehler

\section{Mit Geschlossenheit für die Allergologie}

$\mathrm{N}$ Seit 1992 leite ich den Bereich Allergologie, Berufsdermatologie und Umweltmedizin an der Hautklinik des Universitätsklinikums Münster. Durch den regen Austausch mit Klinikern und Praktikern unterschiedlicher Fachbereiche kenne ich die Möglichkeiten, aber auch die Einschränkungen in der allergologischen Diagnostik und Therapie, die durch Gesetzgebung und Bestimmungen der Krankenkassen unseren Alltag zunehmend beeinflussen.
Eine starke Allergologie setzt die enge Zusammenarbeit und den Schulterschluss zwischen allen in Klinik und Praxis allergologisch tätigen Ärzten voraus.

Die Arbeit des AeDA ist für den Erhalt unseres Faches von grundlegender Bedeutung. Die Tätigkeit im Vorstand des AeDA möchte ich nutzen, um für die Allergologie durch Geschlossenheit Einschränkungen bei der Versorgung von Allergikern entgegenzuwirken und un-

\section{AeDA-Vorstand}

\section{Präsident}

Prof. Dr. Ludger Klimek, Wiesbaden

Vizepräsident

Prof. Dr. Thomas Fuchs, Göttingen

\section{Schatzmeister}

Prof. Dr. Wolfgang W. Schlenter, Frankfurt

\section{Beisitzer}

Prof. Dr. Randolf Brehler, Münster Prof. Dr. Wolfgang Czech, VS-Villingen PD Dr. Kirsten Jung, Erfurt

Prof. Dr. Hans F. Merk, Aachen Norbert K. Mülleneisen, Leverkusen Dr. Katja Nemat, Dresden Dr. Uta Rabe, Treuenbrietzen Prof. Dr. Wolfgang Wehrmann, Münster Dr. Holger Wrede, Herford

\section{Beiräte}

Dr. Bettina Hauswald, Dresden Prof. Dr. Alexander Kapp, Hannover Prof. Dr. Wolfgang Leupold, Dresden Prof. Dr. Dr. Johannes Ring, München

sere Position durch Vernetzung und Verzahnung weiter zu stärken.

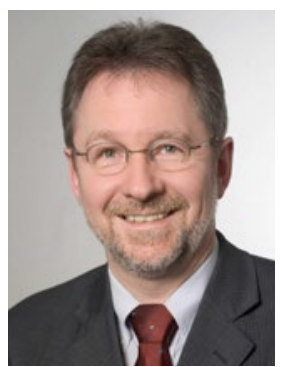

Randolf Brehler

Prof. Dr. Randolf Brehler, Münster

\section{Allergologie in den Köpfen fest verankern}

A ls Facharzt für HNO-Heilkunde, Allergologie, Stimm- und Sprachstörungen bin ich seit zehn Jahren in Herford/Westfalen niedergelassen.

Meine HNO-Facharztausbildung absolvierte ich an der HNO-Klinik Braunschweig und an der HNO-Uniklinik Dresden. Dort lernte ich bei Dr. Bettina Hauswald, Leiterin der Allergieabteilung, was für einen wichtigen Stellen- wert die Allergologie in der HNO-Heilkunde hat, und wie wichtig die interdisziplinäre allergologische Zusammenarbeit ist. Feinschliff erhielt ich anschließend durch Prof. Dr. Ludger Klimek in Wiesbaden.

Für den AeDA möchte ich mich engagieren, um die Allergologie noch fester in den Köpfen der Patienten, der Ärzte in Niederlassung und Kliniken und der Vertreter von Politik und Gesundheitswesen zu verankern.

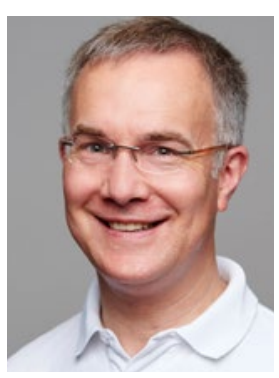

Holger Wrede

Prof. Dr. Holger Wrede, Herford 